\title{
Preoperative total pulmonary blood flow predicts right ventricular pressure in patients early after complete repair of tetralogy of Fallot and pulmonary atresia with major aortopulmonary collateral arteries
}

\author{
Lars Grosse-Wortmann, MD, ${ }^{\mathrm{a}, \mathrm{b}}$ Shi-Joon Yoo, MD, PhD, ${ }^{\mathrm{a}, \mathrm{b}}$ Glen van Arsdell, MD, ${ }^{\mathrm{a}}$ Devin Chetan, HBA, ${ }^{\mathrm{a}}$ \\ Cathy Macdonald, MD, ${ }^{\mathrm{b}}$ Lee Benson, $\mathrm{MD},{ }^{\mathrm{a}}$ and Osami Honjo, $\mathrm{MD}, \mathrm{PhD}^{\mathrm{a}}$
}

\begin{abstract}
Objective: The management of patients with tetralogy of Fallot $(\mathrm{ToF})$ and pulmonary atresia (PA) with major aortopulmonary collateral arteries (MAPCAs) is challenging. Frequently it is difficult to predict whether complete repair with closure of the ventricular septal defect (VSD) will be tolerated. The aim of this study was to investigate whether measurements of preoperative pulmonary blood flow are associated with early postoperative outcome after VSD closure.
\end{abstract}

\begin{abstract}
Methods: In this retrospective study, the data from 10 patients who had undergone a cardiac magnetic resonance imaging study with flow measurements before attempted surgical complete repair were collected. Systemic blood flow (Qs) was calculated as the sum of descending aortic blood flow distal to the MAPCA origins and superior vena cava flow. Pulmonary blood flow (Qp) was measured either from the sum of the pulmonary flow $(n=7)$ or calculated as the difference between ascending aortic flow and Qs.
\end{abstract}

Results: Preoperative Qp/Qs averaged $1.71 \pm 0.68$ and correlated inversely with right ventricular systolic pressure (relative to systolic blood pressure, $r=-0.75, P=.03$ ) and positively with the total neopulmonary artery index (TNPAI, $r=0.66, P=.04$ ), as measured by fluoroscopic angiography. Two children did not tolerate VSD closure. This was likely related to bronchial compression in 1 patient while the other had the lowest TNPAI and the lowest $\mathrm{Qp} / \mathrm{Qs}$ of all patients.

Conclusions: CMR provides not only anatomic but also functional information for surgical decision making in patients with ToF and PA with MAPCAs. Preoperative Qp/Qs is associated with postoperative right ventricular pressure and may be a marker of readiness for VSD closure. (J Thorac Cardiovasc Surg 2013;146:1185-90)

\begin{abstract}
Management of children with tetralogy of Fallot (ToF) and pulmonary atresia (PA) with major aortopulmonary collateral arteries (MAPCAs) has 2 principal goals: (1) to unifocalize the pulmonary blood supply and connect it to the right ventricular outflow tract and (2) to close the ventricular septal defect (VSD), baffling the left ventricular outflow tract to the aortic valve. Strategies to achieve these goals must be individualized to match each child's unique anatomy of pulmonary blood supply. It has been hypothesized that the combined size of all arteries supplying the lungs, including those arising from the aorta and its branches, is a better predictor of postoperative outcome and ability to close the VSD than the number of lung segments supplied by the so-called "native" pulmonary arteries. ${ }^{1}$ In part because of the heterogenous nature of the pulmonary arterial supply,

\footnotetext{
From the Department of Paediatrics, ${ }^{\text {a }}$ The Labatt Family Heart Centre, and the Department of Diagnostic Imaging, ${ }^{\mathrm{b}}$ The Hospital for Sick Children, University of Toronto, Toronto, Ontario, Canada.

Disclosures: Authors have nothing to disclose with regard to commercial support.

Received for publication Sept 22, 2012; revisions received Dec 15, 2012; accepted for publication Jan 14, 2013; available ahead of print Feb 14, 2013.

Address for reprints: Lars Grosse-Wortmann, MD, The Labatt Family Heart Centre, The Hospital for Sick Children, 555 University Ave, Toronto, Ontario, Canada M5G 1X8 (E-mail: lars.grosse-wortmann@sickkids.ca). $0022-5223 / \$ 36.00$

Copyright (c) 2013 by The American Association for Thoracic Surgery http://dx.doi.org/10.1016/j.jtcvs.2013.01.032
}

purely anatomic markers appear not to be optimally suited to predict subsequent right ventricular pressures after VSD closure. ${ }^{2}$ Cardiac magnetic resonance imaging (CMR) has been used to aid in surgical decision making in a number of complex congenital heart defect entities, including patients with aortopulmonary collaterals. ${ }^{3-5}$ It has been used to image MAPCAs but has never been reported in the assessment of pulmonary blood flow in this setting. ${ }^{6-11}$ The aim of the current study was to assess whether total preoperative pulmonary blood flow is a predictor of postoperative outcome after complete repair, including closure of the VSD. The decisionmaking process in a clinical case is described to illustrate how functional CMR can be used to plan a therapeutic strategy for the individual child's anatomy and physiology.

\section{METHODS}

After approval from our institution's research ethics committee, we reviewed the imaging results, demographics, and surgical details of 10 consecutive patients who had had a CMR study before complete repair of ToF and PA with MAPCAs, including attempted VSD closure. The majority of children in this study had been previously studied for another objective. ${ }^{2}$ Patients with an intervention between CMR and complete repair were excluded. Examinations were performed on a 1.5-T General Electric magnet ("Signa CV/I"; General Electric Medical Systems, Milwaukee, Wis) or a 1.5-T Siemens scanner ("Avanto"; Siemens Medical Solutions,
} 


\section{Abbreviations and Acronyms \\ CMR = cardiac magnetic resonance imaging \\ MAPCAs $=$ major aortopulmonary collateral arteries \\ PA $=$ pulmonary atresia \\ PC $=$ as phase-contrast flow velocity mapping \\ PVR = pulmonary vascular resistance \\ $\mathrm{Qp} \quad=$ pulmonary blood flow \\ Qs $\quad=$ systemic blood flow \\ TNPAI = total neopulmonary artery index \\ ToF $\quad=$ tetralogy of Fallot \\ VSD $=$ ventricular septal defect}

Erlangen, Germany). The clinical imaging protocol included static white blood localizer views in 3 orthogonal body planes, cine imaging for ventricular function assessments, as well as phase-contrast flow velocity mapping (PC) of the native pulmonary arteries and veins, the MAPCAs where technically feasible, the aorta proximal and distal to the origin of each MAPCA, and of the superior and inferior venae cavae. From the PC CMR acquisitions, flow volumes were calculated on a commercially available workstation (Qflow 5.2; Medical Imaging Systems, Leiden, The Netherlands). Systemic blood flow (Qs) was measured as the sum of superior vena cava and descending aortic (distal to the MAPCA origins) flows. Pulmonary blood flow $(\mathrm{Qp})$ was measured as the sum of pulmonary venous return. If this was not available it was calculated by subtracting Qs from the ascending aortic flow. Contrast-enhanced magnetic resonance angiography was carried out in the routine fashion in the coronal plane using a bolustracking technique. The 3-dimensional angiographic datasets were reformatted using 1 of 2 commercially available offline workstations ("Advantage Windows 4.2"; General Electric Medical Systems, or "Leonardo"; Siemens Medical Solutions).

In addition to CMR, all patients underwent cardiac catheterization before their full repair. On the basis of the fluoroscopic angioraphy and the operative report, the number of pulmonary segments with vascular supply (either through native pulmonary arteries or unifocalized MAPCAs) was determined. The native pulmonary artery and MAPCA cross-sectional areas were determined from fluoroscopic angiography and summated to calculate the total neopulmonary artery index (TNPAI). ${ }^{12}$ Clinical outcomes were obtained at the time of last clinical follow-up.

\section{Surgical Strategy and Technique}

Details of the surgical strategy and technique are described elsewhere. ${ }^{4}$ Our approach is to perform 1-stage complete unifocalization and VSD closure unless there is distal MAPCA stenosis. Diminutive central pulmonary arteries are initially rehabilitated with an autologous main pulmonary artery-aorta central shunt or a modified Blalock-Taussig shunt before unifocalization. At the time of complete repair, an intraoperative flow study is carried out by inserting an arterial cannula into the reconstructed branch pulmonary arteries. Via this cannula, incremental flow rates of up to 2.5 $\mathrm{L} \cdot \mathrm{min}^{-1} \cdot \mathrm{m}^{-2}$ of body surface area are delivered while the mean pressure in the central pulmonary arteries is monitored. VSD closure is attempted if the mean pulmonary artery pressure does not exceed $30 \mathrm{~mm} \mathrm{Hg}$ during the flow study, among other criteria, including MAPCA and pulmonary arterial morphology. After VSD closure, estimated right ventricular systolic pressure obtained from the maximum tricuspid regurgitation velocity on transesophageal echocardiography and simultaneous systemic systolic blood pressure were recorded.

\section{Statistical Analysis}

Results are presented as means and standard deviation. Pearson correlation analysis was performed to assess the association between parameters of preoperative status and postoperative outcome.

\section{RESULTS}

The mean age at CMR was 0.6 years ( 3 days to 15.3 years, Table 1), and weight was $6.2 \mathrm{~kg}(2.0-55.4 \mathrm{~kg})$. The patients' preoperative, intraoperative, and postoperative investigations are listed in Table 1. Pulmonary arterial blood supply was heterogeneous, reflecting the broad anatomic spectrum in this disease. Eight children had not undergone prior intervention. One patient had had unifocalization of the left-sided MAPCAs into a pericardial pouch that was supplied by a modified Blalock-Taussig while the right lung was supplied by a single MAPCA. Another patient had undergone separate ipsilateral unifocalization procedures of the right and left MAPCAs, supplied via a Blalock-Taussig shunt and a Mee shunt, respectively, before CMR. In 8 children, the VSD was closed. In 1 (patient 2) of the 2 patients who did not have closure of the VSD, the intraoperative flow study revealed a mean pulmonary arterial pressure of $30 \mathrm{~mm} \mathrm{Hg}$ at a flow rate of $2.5 \mathrm{~L} \cdot \mathrm{min}^{-1} \cdot \mathrm{m}^{-2}$. This patient had the lowest $\mathrm{Qp} /$ Qs and lowest TNPAI (Table 1). In the other patient (patient 8) the pulmonary arterial pressure was $25 \mathrm{~mm} \mathrm{Hg}$, but the patient was unable to be weaned from cardiopulmonary bypass and a decision was made to fenestrate the VSD. Retrospectively, the inability to close the VSD was thought to be at least in part due to significant airway problems, including compression of the right main bronchus, requiring airway stenting. The patient died after sepsis and dislodgment of the bronchial stent. One other patient (patient 5), who had undergone VSD closure despite a borderline intraoperative flow study with a mean pulmonary artery pressure greater than $30 \mathrm{~mm} \mathrm{Hg}$, died 13 months after the operation after deterioration of biventricular systolic and diastolic function and multiple chest infections. In all but 2 patients an atrial communication was either created or left patent at the time of complete repair.

Pulmonary blood flow was calculated as the total pulmonary venous return in 7 patients and by subtracting superior vena cava and descending aortic flows from ascending aortic flow in 3. Mean Qp/Qs by CMR was $1.71 \pm 0.68(0.89$ 2.72) and correlated with TPNAI $(r=0.66, P=.038$, Figure 1). In patients who underwent VSD closure, Qp/Qs before complete repair correlated inversely with the intraoperative right ventricular systolic pressure relative to systolic blood pressure after VSD closure $(r=-0.75$, $P=.03$, Figure 2 ). The correlation with absolute right ventricular systolic pressure was not significant. TNPAI correlated inversely with right ventricular systolic pressure $(r=-0.79, P=.02$, Figure 3$)$. The number of pulmonary segments that received arterial supply at the time of 
complete repair did not predict intraoperative right ventricular systolic pressure. Neither TNPAI, number of vascularized pulmonary segments, nor Qp/Qs correlated with the pulmonary arterial pressures during the intraoperative flow study. Preoperative oxygen saturations did not correlate with preoperative $\mathrm{Qp} / \mathrm{Qs}$ or TNPAI. The intraoperative flow study results or preoperative oxygen saturations did not correlate with absolute or relative intraoperative right ventricular systolic pressures.

\section{Clinical Scenario}

A 14-year-old girl (patient 7, Table 1) with unrepaired ToF and PA with MAPCAs was referred for CMR and cardiac catheterization for anatomic and hemodynamic evaluation. Contrast-enhanced magnetic resonance angiography demonstrated a single large MAPCA to the left lung with midsegment stenosis as well as 2 closely spaced MAPCAs from the descending aorta to the right lung (Figure 4). Phase contrast flow velocity mapping measured $3.61 \mathrm{~L} \cdot \mathrm{min}^{-1}$. $\mathrm{m}^{-2}$ ( $63 \%$ of total pulmonary blood flow) of blood flow to the left lung and $2.16 \mathrm{~L} \cdot \mathrm{min}^{-1} \cdot \mathrm{m}^{-2}$ to the right lung (37\% of pulmonary blood flow). Flow in the right MAPCAs showed a hypertensive flow profile; the pattern in the left MAPCA distal to the stenosis was continuous with high velocity (Figure 4). These blood flows were interpreted in combination with invasive pressure measurements to yield a selective pulmonary vascular resistance (PVR) of 0.6 $\mathrm{WU} \cdot \mathrm{m}^{2}$ on the left and $21.3 \mathrm{WU} \cdot \mathrm{m}^{2}$ on the right. Fourteen years of unprotected systemic arterial blood flow to the right lung had led to advanced pulmonary vascular disease in the affected lung, whereas the contralateral left lung had been protected by the narrowing within the supplying vessel. The patient underwent complete repair with unifocalization of the MAPCAs and VSD closure. Six days postoperatively, the right lung perfusion ratio had further decreased from $37 \%$ to $14 \%$ of total pulmonary blood flow, as measured by CMR, reflecting persistent pulmonary vascular changes from unprotected pulmonary blood flow on the right.

\section{DISCUSSION}

The principal findings of this study are as follows:

1. Total pulmonary blood flow in patients with ToF and PA with MAPCAs can be measured using CMR

2. Qp/Qs correlates inversely with right ventricular pressure after VSD closure

3. TNPAI correlates with $\mathrm{Qp} / \mathrm{Qs}$ before the operation and inversely with right ventricular pressure after VSD closure

In patients with ToF and PA with MAPCAs, the goals of pulmonary artery reconstruction are (1) the recruitment of a maximum number of alveolae into the pulmonary circulation for gas exchange and (2) the creation of a lowresistance vascular bed to receive the total right ventricular cardiac output, making it possible to septate the ventricles. Strategies toward both goals include stimulating growth of the intrapericardial ("native") pulmonary arteries via an aortopulmonary shunt or ductal stenting and/or to connect the maximum number of MAPCAs to the right ventricular outflow tract. Whether or not a patient is ready for VSD closure is difficult to predict in clinical practice. "Borderline" patients face the risk of right ventricular hypertension and failure after complete repair with VSD closure. On the other hand, a potentially unnecessary staging of the repair, deferring VSD closure into the future, carries the burden of additional interventions and a persistent shunt across the VSD. ${ }^{12}$ The status of the pulmonary vascular bed has been identified as a crucial determinant of successful complete repair. ${ }^{1}$ Ultimately, the ability to close the VSD is a function of total PVR, which dictates pulmonary blood flow in light of a finite pressure that the right ventricle is able to generate. Consequently, if the combined PVR of both lungs is known preoperatively, it should be possible to predict whether closure of the VSD will be tolerated, provided that ventricular function is normal and that there is no proximal pulmonary artery obstruction. Traditionally, anatomic information, obtained either by direct inspection or fluoroscopic angiography, has been used to characterize the status of the patient's pulmonary vasculature. The Nakata, McGoon, and total neopulmonary artery indices are attempts to quanitify this status, assuming that arterial size and cross-sectional area are predictive of total PVR. ${ }^{13}$ In fact, TNPAI correlated with right ventricular systolic pressures after VSD closure, supporting the notion that the total cross-sectional area of the vessels supplying the lungs and not necessarily their embryologic origin as either MAPCAs or native pulmonary arteries is a key determinant of postoperative pulmonary artery pressure. ${ }^{1,13-16}$

Although it is intuitive that TNPAI yields information about the pulmonary vascular bed, the principal limitation of vessel size indices is that pulmonary vascular physiology is not directly assessed but merely inferred from morphologic appearance. Furthermore, TNPAI does not take into account peripheral stenoses and pulmonary vascular changes that are common in this condition. $\mathrm{We}^{2}$ were recently able to show that a functional parameter-central pulmonary pressure in response to a standardized flow via cardiopulmonary bypass after VSD closure-was a more important predictor of postoperative outcomes than TNPAI. In line with these results, the Stanford group found that the use of routine intraoperative flow studies led to fewer cases in which the VSDs had to be reopened. ${ }^{1}$ One fundamental benefit of intraoperative flow studies is that it takes into account the technical adequacy of the repair. Despite the usefulness of intraoperative flow studies, it would have obvious advantages to have functional information on the pulmonary vascular bed before the operation: the information would be available for surgical decision making and 
TABLE 1. Demographic, surgical and imaging data

\begin{tabular}{|c|c|c|c|c|c|c|}
\hline $\begin{array}{l}\text { Patient } \\
\text { No. } \\
\end{array}$ & $\begin{array}{c}\text { Age at } \\
\operatorname{CMR}(y)\end{array}$ & $\begin{array}{l}\mathrm{O}_{2} \text { saturation } \\
\text { at } \mathrm{CMR}(\%)\end{array}$ & $\begin{array}{c}\text { Qp }\left(L \cdot \min ^{-1} \cdot \mathrm{m}^{-2}\right) \\
\text { by CMR } \\
\end{array}$ & $\begin{array}{c}\text { Qp/Qs } \\
\text { by CMR }\end{array}$ & $\begin{array}{c}\text { TNPAI }\left(\mathrm{mm}^{2} / \mathrm{m}^{2}\right) \\
\text { by fluoroscopic angiography }\end{array}$ & $\begin{array}{c}\text { Age at complete } \\
\text { repair }(y)\end{array}$ \\
\hline 1 & 15.3 & 82 & 5.51 & 1.30 & 95.7 & 16.2 \\
\hline 2 & 0.5 & 81 & 4.5 & 0.89 & 62.8 & 1.6 \\
\hline 3 & 1.0 & 78 & 4.73 & 1.23 & 180.8 & 1.3 \\
\hline 4 & 0.6 & 70 & 4.34 & 2.11 & 333.7 & 0.7 \\
\hline 5 & 0.9 & 80 & 7.38 & 2.72 & 174.0 & 1.0 \\
\hline 6 & $\begin{array}{l}0.0 \\
7 \mathrm{~d}\end{array}$ & 88 & 4.96 & 1.06 & 164.0 & 4.0 \\
\hline 7 & 14.1 & 83 & 6.2 & 2.58 & 297.0 & 15.0 \\
\hline 8 & 0.2 & 80 & 4.1 & 1.37 & 162.3 & 0.5 \\
\hline 9 & 2.4 & 90 & 4.72 & 0.94 & 149.8 & 2.6 \\
\hline 10 & $\begin{array}{l}0.0 \\
3 \mathrm{~d}\end{array}$ & 95 & 3.46 & 1.58 & 132.5 & 0.5 \\
\hline
\end{tabular}

$C M R$, Cardiac magnetic resonance; $O_{2}$, oxygen; $Q p$, pulmonary blood flow; $Q s$, systemic blood flow; TNPAI, total neopulmonary artery index; $P A$, pulmonary arteries; $V S D$, ventricular septal defect; $R V s p$, right ventricular systolic pressure; $B P s y s$, systolic blood pressure; $S D$, standard deviation. *At a flow rate of $2.5\left(\mathrm{~L} \cdot \mathrm{min}^{-1} \cdot \mathrm{m}^{-2}\right)$ of cardiac bypass flow. †Inability to close the VSD was believed to be in part due to severe obstruction of the right main bronchus.

procedural planning, and the conditions would be more representative of the true physiology than on cardiopulmonary bypass with suboptimally expanded lungs.

One of the major strengths of CMR is the ability to provide functional information, ${ }^{17,18}$ which has allowed us to tighten the margins of error in surgical decision making in complex congenital heart disease. ${ }^{4}$ Blood flow measurements, in particular, have proven to be a great asset in the evaluation of complex shunt lesions. ${ }^{3,19-21}$ Flow evaluation by CMR has distinct advantages over other functional assessments: in contrast to cardiac catheterization, it is noninvasive (except for the sedation or anesthesia that is often required when examining neonates and infants with magnetic resonance) and uses no ionizing radiation. These properties make CMR a suitable tool for serial follow-up examinations in patients who undergo interventions with the goal to recruit additional pulmonary vasculature, to promote growth of the central pulmonary arteries, and/or to lower PVR before complete repair.

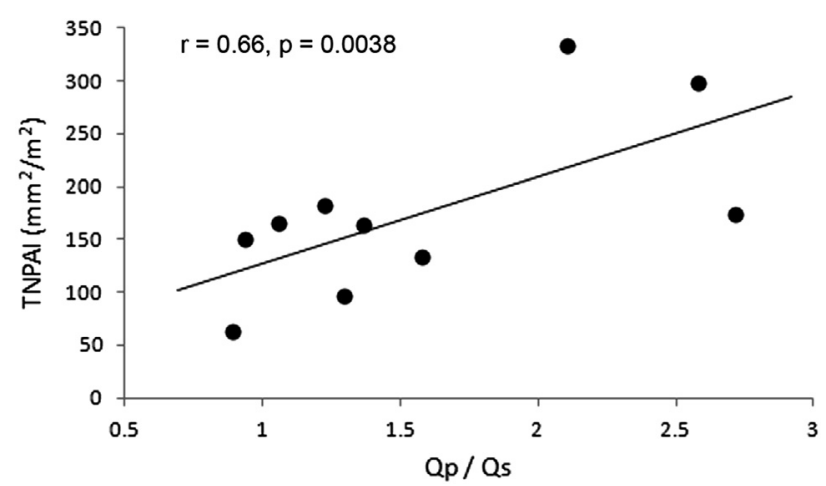

FIGURE 1. Correlation between preoperative total to pulmonary/systemic blood flow ratio $(Q p / Q s)$ and preoperative total neopulmonary artery index (TNPAI).
In patients with ToF and PA with MAPCAs, pulmonary blood flow can be obtained using PC CMR using 1 of 3 strategies: (1) direct measurement of MAPCA flow, (2) subtraction of aortic blood flow distal from flow proximal to the MAPCA origin(s), or by (3) the sum of pulmonary venous return. Particularly in patients with complex MAPCA anatomy, option 3 is technically easiest. The accuracy of PC measurements of pulmonary venous flow has been demonstrated. ${ }^{18,21} \mathrm{We}^{3}$ and others ${ }^{22}$ have used this technique to determine aortopulmonary collateral flow in small children with single ventricle physiology.

In the cohort studied here, CMR derived pulmonary blood flow (relative to systemic blood flow) predicted intraoperative right ventricular systolic pressures after VSD closure (relative to systolic blood pressure). The link between the 2 is total PVR, inasmuch as both parameters are functions of total PVR (in the absence of outflow tract and pulmonary artery obstruction). In fact, PVR is defined as the ratio of the pressure gradient across a vascular bed and the flow through it.

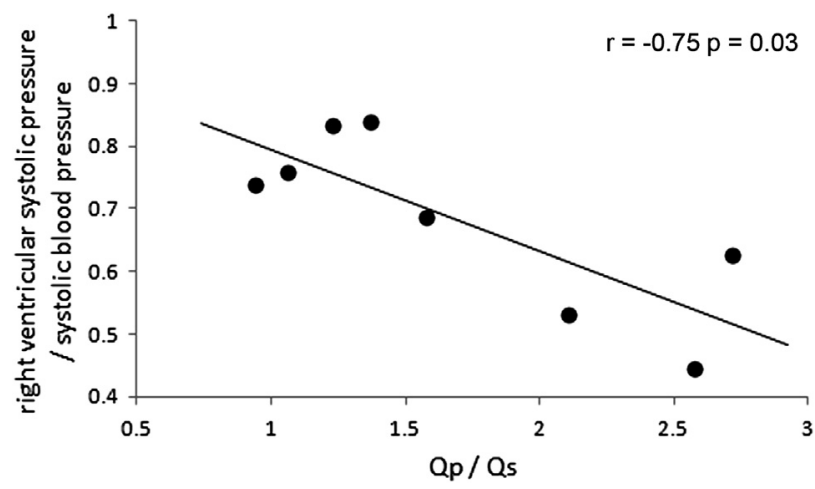

FIGURE 2. Correlation between preoperative total to pulmonary/systemic blood flow ratio $(Q p / Q s)$ and intraoperative right ventricular systolic pressure, relative to systolic blood pressure. 
TABLE 1. Continued

\begin{tabular}{ccccc}
\hline $\begin{array}{c}\text { PA pressure } \\
\text { during intraoperative } \\
\text { flow study (mm Hg)* }\end{array}$ & $\begin{array}{c}\text { No. of pulmonary } \\
\text { segments that were vascularized } \\
\text { after complete repair }\end{array}$ & VSD closure & $\begin{array}{c}\text { Intraoperative } \\
\text { RVsp (mm Hg) }\end{array}$ & RVsp/BPsys \\
\hline 25 & 14 & Yes & 83 & 0.755 \\
30 & 15 & No & NA & NA \\
23 & 18 & Yes & 63 & 0.833 \\
24 & 16 & Yes & 35 & 0.530 \\
30 & 14 & Yes & 50 & 0.625 \\
17 & 14 & Yes & Yes \\
11 & 18 & Yes & 62 & 0.756 \\
25 & 18 & No $\dagger$ & 40 & 0.444 \\
15 & 18 & Yes & NA & Yes \\
13 & 15 & Yes & 53 & Nes \\
& & & 52 & 0.736 \\
\end{tabular}

In simple left-to-right shunt lesions, pulmonary blood flow can be used to predict PVR. ${ }^{19}$ Longstanding exposure of the pulmonary vascular bed to high flow and systemic pulsatile perfusion pressure can result in irreversible pulmonary hypertensive changes, as illustrated by our clinical case. Stenosis along the MAPCA course, on the other hand, may protect the dependent lung segments from irreversible damage. Therefore, it seems reasonable to speculate that a high preoperative $\mathrm{Qp} / \mathrm{Qs}$ is reflective of unobstructed pulmonary blood flow and a compliant vascular bed and, therefore, conveys good candidacy for VSD closure. A low $\mathrm{Qp} / \mathrm{Qs}$, on the other hand, warrants further investigation to differentiate between small vessel disease and proximal stenosis. For detailed surgical planning, not only the total, but also the unilateral PVR indices for each lung, or even each territory supplied by a defined MAPCA, are of interest. The quantification of unilateral PVR requires selective pressure as well as flow measurements. Indicator dilution techniques such as the Fick method to measure blood flow are not applicable here because the oxygen

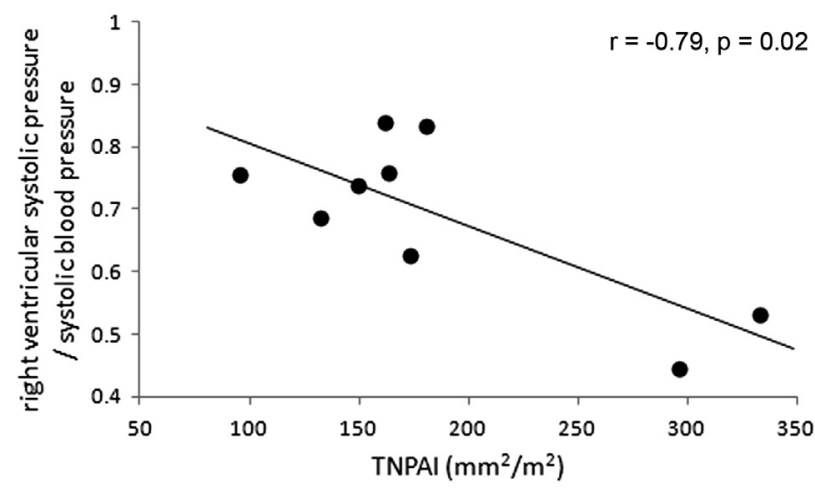

FIGURE 3. Correlation between preoperative total neopulmonary artery index (TNPAI) and intraoperative right ventricular systolic pressure after ventricular septal defect closure, relative to systolic blood pressure. uptake in each lung cannot be assessed independently of the other lung. The combination of invasive manometry and flow measurements by PC CMR described here is the only clinically feasible method to measure unilateral $\mathrm{PVR}^{20}$ To our knowledge, this is the first reported case of unilateral PVR calculation in a clinical setting, using PC CMR for flow assessment.

\section{Limitations}

Several limitations pertaining to this study are worth mentioning: the cohort size was small, limiting statistical power. Further, the interval between CMR and complete repair was considerable in some patients. Qp, if measured as the total pulmonary venous return, falsely includes a contribution from the bronchial arteries, thought to be about $6 \%$ of cardiac output. Qs, on the other hand, does not take into consideration the blood flow in the coronary arteries. However, these deviations from true Qp and Qs, respectively, are thought to be lower than the measurement inaccuracies of PC CMR.

\section{CONCLUSIONS}

CMR provides not only anatomic but also functional information for surgical decision making in children with ToF and PA with MAPCAs. Pulmonary blood flow in patients with this anomaly can be quantified by CMR. Preoperative Qp/Qs correlates with post-VSD closure RV systolic pressures. For a comprehensive and selective evaluation of preoperative PVR, invasive pressure measurements ideally are combined with CMR flow measurements. We anticipate that this approach will be suited to contribute to patient selection for complete repair, but prospective studies in a larger cohort are needed to test its ability to predict long-term outcome after complete repair. 

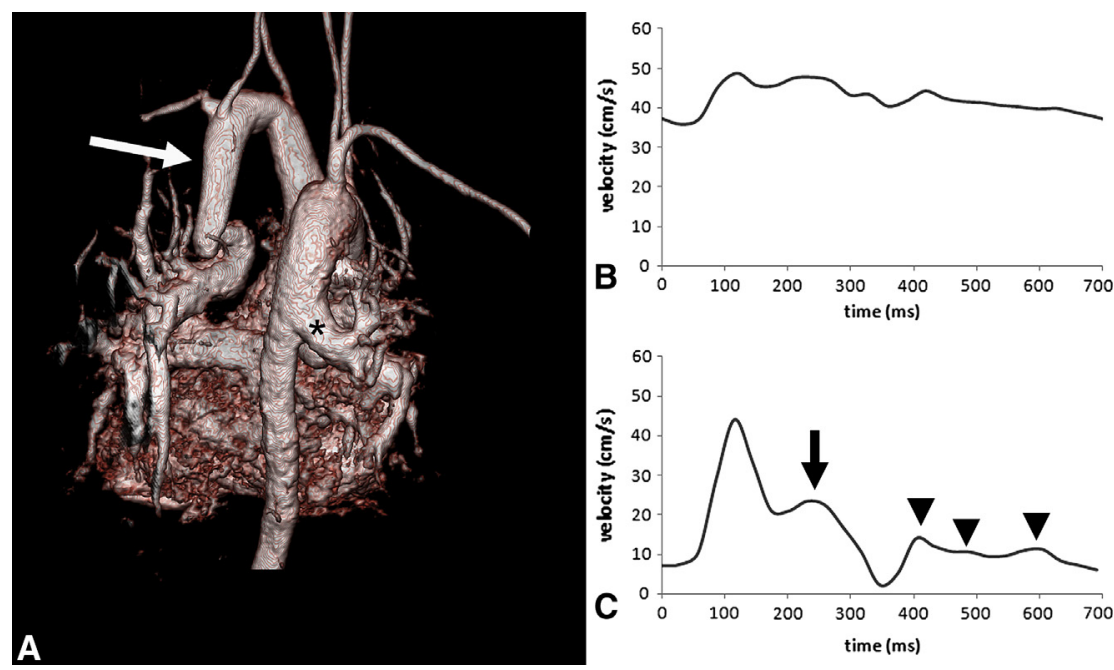

FIGURE 4. A, The volume rendered contrast-enhanced magnetic resonance angiography shows a tortuous obstructed major aortopulmonary collateral artery (MAPCA) to the left lung, arising from the left subclavian artery (arrow) and a widely patent MAPCA to the right lung from the descending aorta (asterisk), seen from posterior. Flow velocity profiles in the left (B) and right (C) MAPCAs. The left MAPCA shows a poststenotic flow profile with high velocity continuous flow whereas the flow profile in the right MAPCA exhibits hypertensive changes, including an early systolic peak, an accessory peak in late systole (arrow), and an undulating baseline with multiple peaks (arrowheads) throughout diastole.

\section{References}

1. Reddy VM, McElhinney DB, Amin Z, Moore P, Parry AJ, Teitel DF, et al. Early and intermediate outcomes after repair of pulmonary atresia with ventricular septal defect and major aortopulmonary collateral arteries: experience with 85 patients. Circulation. 2000;101:1826-32.

2. Honjo O, Al-Radi OO, MacDonald C, Tran KC, Sapra P, Davey LD, et al. The functional intraoperative pulmonary blood flow study is a more sensitive predictor than preoperative anatomy for right ventricular pressure and physiologic tolerance of ventricular septal defect closure after complete unifocalization in patients with pulmonary atresia, ventricular septal defect, and major aortopulmonary collaterals. Circulation. 2009;120:S46-52.

3. Grosse-Wortmann L, Al-Otay A, Yoo SJ. Aortopulmonary collaterals after bidirectional cavopulmonary connection or Fontan completion: quantification with MRI. Circ Cardiovasc Imaging. 2009;2:219-25.

4. Grosse-Wortmann L, Yun TJ, Al-Radi O, Kim S, Nii M, Lee KJ, et al. Borderline hypoplasia of the left ventricle in neonates: insights for decision-making from functional assessment with magnetic resonance imaging. J Thorac Cardiovasc Surg. 2008;136:1429-36.

5. Grosse-Wortmann L, Drolet C, Dragulescu A, Kotani Y, Chaturvedi R, Lee KJ, et al. Aortopulmonary collateral flow volume affects early postoperative outcome after Fontan completion: a multimodality study. $J$ Thorac Cardiovasc Surg. 2012;144:1329-36.

6. Durongpisitkul K, Saiviroonporn P, Soongswang J, Laohaprasitiporn D, Chanthong P, Nana A. Pre-operative evaluation with magnetic resonance imaging in tetralogy of Fallot and pulmonary atresia with ventricular septal defect. J Med Assoc Thai. 2008;91:350-5.

7. Rees RS, Somerville J, Underwood SR, Wright J, Firmin DN, Klipstein RH, et al. Magnetic resonance imaging of the pulmonary arteries and their systemic connections in pulmonary atresia: comparison with angiographic and surgical findings. Br Heart J. 1987;58:621-6.

8. Geva T, Greil GF, Marshall AC, Landzberg M, Powell AJ. Gadolinium-enhanced 3-dimensional magnetic resonance angiography of pulmonary blood supply in patients with complex pulmonary stenosis or atresia: comparison with x-ray angiography. Circulation. 2002;106:473-8.

9. Kawel N, Valsangiacomo-Buechel E, Hoop R, Kellenberger CJ. Preoperative evaluation of pulmonary artery morphology and pulmonary circulation in neonates with pulmonary atresia - usefulness of MR angiography in clinical routine. J Cardiovasc Magn Reson. 2010;12:52.

10. Powell AJ, Chung T, Landzberg MJ, Geva T. Accuracy of MRI evaluation of pulmonary blood supply in patients with complex pulmonary stenosis or atresia. Int J Card Imaging. 2000;16:169-74.

11. Vick GW III, Wendt RE III, Rokey R. Comparison of gradient echo with spin echo magnetic resonance imaging and echocardiography in the evalua- tion of major aortopulmonary collateral arteries. Am Heart J. 1994;127: 1341-7.

12. Reddy VM, Petrossian E, McElhinney DB, Moore P, Teitel DF, Hanley FL. Onestage complete unifocalization in infants: when should the ventricular septal defect be closed? J Thorac Cardiovasc Surg. 1997;113:858-66.

13. Amark KM, Karamlou T, O'Carroll A, MacDonald C, Freedom RM, Yoo SJ, et al. Independent factors associated with mortality, reintervention, and achievement of complete repair in children with pulmonary atresia with ventricular septal defect. J Am Coll Cardiol. 2006;47:1448-56.

14. Cho JM, Puga FJ, Danielson GK, Dearani JA, Mair DD, Hagler DJ, et al. Early and long-term results of the surgical treatment of tetralogy of Fallot with pulmonary atresia, with or without major aortopulmonary collateral arteries. $J$ Thorac Cardiovasc Surg. 2002;124:70-81.

15. Davies B, Mussa S, Davies P, Stickley J, Jones TJ, Barron DJ, et al. Unifocalization of major aortopulmonary collateral arteries in pulmonary atresia with ventricular septal defect is essential to achieve excellent outcomes irrespective of native pulmonary artery morphology. $J$ Thorac Cardiovasc Surg. 2009;138: 1269-75.

16. Song SW, Park HK, Park YH, Cho BK. Pulmonary atresia with ventricular septal defects and major aortopulmonary collateral arteries. Circ J. 2009;73: 516-22.

17. Kellenberger CJ, Macgowan CK, Roman KS, Al-Habshan F, Benson LN, Redington AN, et al. Hemodynamic evaluation of the peripheral pulmonary circulation by cine phase-contrast magnetic resonance imaging. J Magn Reson Imaging. 2005;22:780-7.

18. Grosse-Wortmann L, Al-Otay A, Goo HW, Macgowan CK, Coles JG, Benson LN, et al. Anatomical and functional evaluation of pulmonary veins in children by magnetic resonance imaging. J Am Coll Cardiol. 2007;49:993-1002.

19. Bell A, Beerbaum P, Greil G, Hegde S, Toschke AM, Schaeffter T, et al. Noninvasive assessment of pulmonary artery flow and resistance by cardiac magnetic resonance in congenital heart diseases with unrestricted left-to-right shunt. JACC Cardiovasc Imaging. 2009;2:1285-91.

20. Muthurangu V, Taylor A, Andriantsimiavona R, Hegde S, Miquel ME, Tulloh R, et al. Novel method of quantifying pulmonary vascular resistance by use of simultaneous invasive pressure monitoring and phase-contrast magnetic resonance flow. Circulation. 2004;110:826-34.

21. Goo HW, Al-Otay A, Grosse-Wortmann L, Wu S, Macgowan CK, Yoo SJ. Phasecontrast magnetic resonance quantification of normal pulmonary venous return. J Magn Reson Imaging. 2009;29:588-94.

22. Whitehead KK, Gillespie MJ, Harris MA, Fogel MA, Rome JJ. Noninvasive quantification of systemic-to-pulmonary collateral flow: a major source of inefficiency in patients with superior cavopulmonary connections. Circ Cardiovasc Imaging. 2009;2:405-11. 